\title{
Estimation of the Pulmonary Elastance Using a Recursive Parameter Estimation Method and Setting of the Ventilation Condition Using Fuzzy Logic*
}

\author{
Masanori NAKAMICHI ${ }^{\dagger}$ and Shunshoku $\mathrm{KANAE}^{\dagger}$
}

\begin{abstract}
Artificial respirators are widely used for patients with little or no autonomous breathing ability. Doctors are required to pay scrupulous attention for the use of the artificial respirators. And doctors must set the artificial respirator in consideration of each patient's pulmonary characteristic. However, the setting of the artificial respirator is decided by the experience and the intuition of the doctor now. The purpose of this study is to develop a method to estimate the static $P-V$ curve and the pulmonary elastance of the patient and to set a ventilation condition of the artificial respirator. In our previous work, we have presented an estimation technique of the pulmonary elastance by fuzzy logic. By improving previous technique, this study succeeded in improving the precision of the pulmonary elastance estimation. In addition, a ventilation condition of the artificial respirator is set using estimated static $P-V$ curve and fuzzy logic.
\end{abstract}

\section{Introduction}

Artificial respirators are widely used for patients with little or no autonomous breathing ability. Doctors are required to pay scrupulous attention for the use of the artificial respirators (the excessive air flow or the excessive pressure). And doctors must set the artificial respirator in consideration of each patient's pulmonary characteristic. However, there are two following reasons to obstruct setting the artificial respirator in consideration of patient's pulmonary characteristic. The first problem is that the characteristic of human lungs is different and changes by state of disease. The data which can be measured in the artificial respirator are only of the air-way opening pressure and the air-flow. These data do not directly express the characteristic of the human lungs such as pulmonary internal pressure. Therefore, we do not understand the pulmonary characteristic of the patient by the measurement of data. The second problem is that the setting of the artificial respirator is decided by the experience and the intuition of the doctor. From the problem mentioned above, the setting method of the artificial respirator suitable for the pulmonary characteristic of the patient does not exist. If there is a patient (a premature baby etc.) who cannot be applied the experience and the intuition of the doctor, what can we do? If there is a patient with no autonomous breathing ability in the town with-

\footnotetext{
* Manuscript Received Date : May 20, 2014

† Fukui University of Technology; Gakuen, Fukui city, Fukui 910-8505, JAPAN

Key Words: fuzzy logic, pulmonary elastance, ventilation condition.
}

out doctor, what can we do? In the current setting method of the artificial respirator, we can do nothing. Our study is going to solve these problems.

Purpose of this study is to develop a method to estimate the static $P-V$ curve and the pulmonary elastance of the patient and to set a ventilation condition of the artificial respirator[1]. The static $P-V$ curve and the pulmonary elastance expresses the important feature of the lung, and the static $P-V$ curve is a basis for deciding the air-way pressure limit value. However, the static $P-V$ curve cannot be drawn directly, because inside pressure of lung cannot be measured directly. So, we must estimate the static $P-V$ curve by technique of the system identification.

In our previous work, we have presented an estimation technique of the pulmonary elastance by fuzzy logic and have represented the static $P-V$ curve needed for the artificial respirator setting. However, a problem was found in the previous estimation technique. About a fuzzy logic, the pulmonary elastance (nonlinear function) is divided into some fuzzy rules. Then, consequent part parameters of each fuzzy rule are estimated. When those parameters are estimated, the respiratory system model is used. The respiratory system is constructed in the pulmonary elastance term, air-way resistance term, etc. Parameters of the pulmonary elastance $\left(f_{E}(V)\right)$ are different in each fuzzy rules, but other parameters do not change in a short time(one cycle breath). Nevertheless, in the previous study, these parameters were estimated to be different values in parameters estimation of each fuzzy rule. It is considered that the estimated precision of the static $P-V$ curve is influenced by these values. In this study, we solve this problem using new 
estimation procedure. Then, this estimation procedure can repeat itself, and the estimation precision improves repeatedly. In addition, a ventilation condition of the artificial respirator is set using estimated static $P-V$ curve. This setting value is decided using fuzzy logic. There is an advantage that this technique has less computational complexity than existing technique. In this paper, the techniques mentioned above are introduced and the effectiveness of the suggested techniques is illustrated by an estimation example using real patient data.

The paper is organized as follows. Section $\mathbf{2}$ introduces the static $P-V$ curve and nonlinear differential equation model of respiratory systems. A new method that estimating the pulmonary elastance by fuzzy logic is described in Section 3. A method that setting a ventilation condition of the artificial respirator by fuzzy logic is explained in Section 4. Some examples of estimation are given in Section 5. Finally, Section 6 concludes this paper.

\section{2. $P-V$ Curve and Nonlinear Dif- ferential Equation Model of Res- piratory Systems}

In this section, $P-V$ curve and respiratory system equation are explained.

We breathe by flowing air in the respiratory system. This state is dynamic state. The static state is a state without the flow of air in the respiratory system. In this state, the static $P-V$ curve is drawn by the pulmonary internal pressure $\left(P_{\ell}(t)\right)$ and the volume inside of lung $(V(t))$. The gradient of the static $P-V$ curve is the compliance, and the inverse of the compliance is the elastance of the lung. The pulmonary elastance $\left(f_{E}(V)\right)$ is defined as a nonlinear function of $V(t)$, and the static $P-V$ curve is expressed by the following equation:

$$
P_{\ell}=f_{E}(V, \theta) V
$$

This static $P-V$ curve expresses the important feature of the lung. Therefore, we can perform setting of the artificial respirator based on the feature of the lung if we can understand the static $P-V$ curve of the patient. For example, the $P-V$ curve is a basis for deciding the air-way pressure limit value.

Fig.1 shows the dynamic $P-V$ curve (solid line) and the static $P-V$ curve (dotted line) by respiratory data over one cycle breath. The dynamic $P-V$ curve can be drawn by air-way pressure and air-volume of lung that can be measured directly. However, the static $P-V$ curve cannot be drawn directly, because the pulmonary internal pressure $\left(P_{\ell}(t)\right)$ cannot be measured directly. So, we must estimate the static $P-V$ curve by technique of the system identification.

For a rational setting of the artficial respirator, a mathematical model is needed to describe the respiratory system. In dynamics, the basic element of respiratory system is pressure, volume and flow. Then,

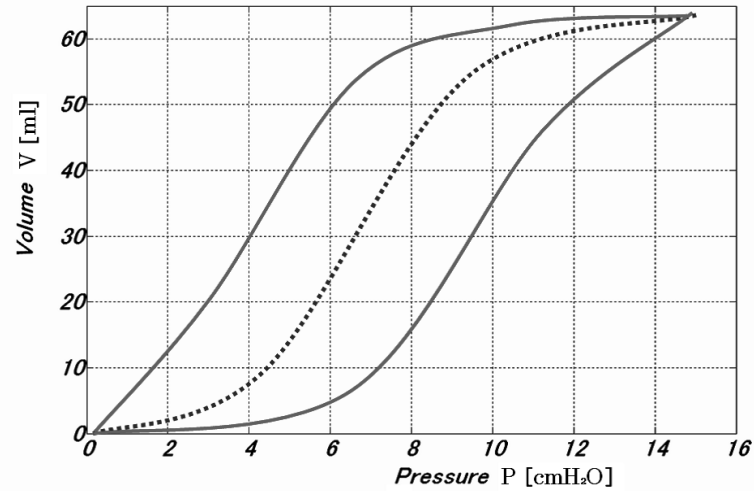

Fig. 1 The dynamical $P-V$ curve (solid line) and the static $P-V$ curve (dotted line)

various resistance (elastance and friction) occurs by air flow. The human being has the right lung and the left lung. Even if each lung is modeled by a simple first order differential equation, the synthesized overall respiratory system model becomes to second order differential equation. Respiratory system model as the following equation is proposed by Kanae et $a l[2][3]$,

$$
\begin{aligned}
P_{a o}(t)+a_{1} \dot{P}_{a o}(t)= & f_{E}(V) V(t)+g_{R}(\dot{V}) \dot{V}(t) \\
& +b_{2} \ddot{V}(t)+P_{\text {eea }}+\epsilon(t),
\end{aligned}
$$

where $P_{a o}(t)$ is air-way opening pressure, $F(t)=\dot{V}(t)$ is air-flow, $P_{\text {eea }}$ is the end-expiratory alveolar pressure. In addition, $g_{R}(\dot{V})$ is so-called air-way resistance. The pressure loss $P_{r}$ is expressed by the following equation,

$$
P_{r}(t)=g_{R}(F) F(t)=\left(r_{1}+r_{2}|\dot{V}(t)|\right) \dot{V}(t) .
$$

The pulmonary elastance and the air-way resistance are described by polynomials of volume $V(t)$. $\epsilon(t)$ contains the modeling error and the measurement noise. In the model (2), the order of equation are the same as the conventional model. Therefore, the model has the capability of describing the nonlinear dynamical characteristics of respiration. We can measure airway opening pressure $P_{a o}(t)$, volume $V(t)$, air-flow $F(t)$ in each sampling period.

The problem is expression of the pulmonary elastance. The polynomial expression and the RBF network expression was suggested so far. However, there are problems in those expression[1]. The fuzzy logic is suitable for expressing a nonlinear function, and can reduce computational complexity in the estimation by the fuzzy division. After that, using this respiratory system model (2), this study presents a method to estimate the pulmonary elastance by fuzzy logic. 


\section{Fuzzy Model of Respiratory Sys- tems}

\subsection{Fuzzy Logic}

Real world has a lot of fuzzy information. These information varies according to subjectivity and they did not have a general standard and law. The fuzzy logic is a logic to valuate these fuzzy information. This logic was suggested by L.A.Zadeh in 1965 and is applied to various fields today[4]. Mamdani's fuzzy IF-THEN rules are very famous in fuzzy logic. The structure of these rules is that all inputs are set in part of the antecedent and all outputs are set in part of the consequent. The fuzzy reasoning is carried out using this rule.

In this study, the volume of the lungs $(V(t))$ is defined as the part of the antecedent because pulmonary elastance value changes at the volume of the lungs. Then, pulmonary elastance of the first order function of $\left(f_{E}(V)=k_{1}+k_{2} V\right)$ is defined as the part of the consequent. Fuzzy variables in part of the antecedent are defined as small, medium, big.

In a previous study, we developed technique to do simple fuzzy rules by combining the data of two phases of expiratory and inspiratory respiration in every same fuzzy variable range[5]. Using this technique, the fuzzy rule is expressed as follows:

$$
\begin{aligned}
& \text { if } V=\text { small, } \\
& \text { then } f_{E(\text { small })}=k_{1(\text { small })}+k_{2(\text { small })} V ; \\
& \text { if } V=\text { medium, } \\
& \text { then } f_{E(\text { medium })}=k_{1(\text { medium })}+k_{2(\text { medium })} V ;(4) \\
& \text { if } V=\text { big, } \\
& \text { then } f_{E(\text { big })}=k_{1(\text { big })}+k_{2(\text { big })} V .
\end{aligned}
$$

Using combined data, the parameters of the consequent part of the fuzzy rules (4) are estimated by the least squares method.

Using above fuzzy rules (4), the reasoning result of pulmonary elastanse is estimated. When input value (the volume of the lung) was given, the fuzzy reasoning is carried out using applicable rules. Please note that the reasoning result is given with numerical value and is not given a fuzzy concept. The reasoning result of fuzzy rules (4) is calculated by the Center of Gravity Method as follows:

$$
\begin{aligned}
& h_{j}^{V}=A_{j}^{V}\left(V^{0}\right), \quad(j=\text { small,medium, big }), \\
& f_{E}^{0}=\frac{\sum_{j=\text { small }}^{\text {big }} h_{j}^{V} f_{E(j)}\left(V^{0}\right)}{\sum_{j=\text { small }}^{\text {big }} h_{j}^{V}},
\end{aligned}
$$

where, $A$ is fuzzy set of the antecedent variable $V, h_{j}$ is the conformity degree of the antecedent. The static $P-V$ curve is drawn by equation (1),

\subsection{Estimation of Consequent Part Pa- rameters of the Fuzzy Rule}

This section introduces the method that estimates the function parameters of consequent part about an arbitrary rule in fuzzy rule of equation (4). Using the respiratory system model, the first order function $\left(f_{E}(V)=k_{1}+k_{2} V\right)$ substitutes the relationship of

$$
\begin{aligned}
P_{a o}(t)+a_{1} \dot{P}_{a o}(t) & =k_{1} V(t)+k_{2} V^{2}(t) \\
& +g_{R}(\dot{V}) \dot{V}(t)+b_{2} \dot{V}(t)+\epsilon(t) .
\end{aligned}
$$

In the previous study, the end-expiratory alveolar pressure $P_{\text {eea }}$ of respiratory system at each fuzzy rule was estimated different value. However, $P_{\text {eea }}$ does not change in short time (one cycle breath). We can estimate $P_{\text {eea }}$ value from patient measurement data (end-expiratory pressure data) easily. Therefore, we remove $P_{\text {eea }}$ term from the respiratory system model before estimation algorithm practice. Define data vector $\varphi(t)$ and parameter vector $\theta$ as follows: $\varphi^{T}(t)=\left[-\dot{P}_{a o}(t), V(t), V^{2}(t), \dot{V}(t),|\dot{V}(t)| \dot{V}(t)\right.$, $\ddot{V}(t)] ; \theta^{T}=\left[a_{1}, k_{1}, k_{2}, r_{1}, r_{2}, b_{2}\right]$. Then, using relationship between the volume and the flow $(F(t)=\dot{V}(t))$, data vector $\varphi(t)$ can be written as follows: $\varphi^{T}(t)=$ $\left[-\dot{P}_{a o}(t), V(t), V^{2}(t), F(t),|F(t)| F(t), \dot{F}(t)\right]$.

The model equation can also be written in short form as follows:

$$
P_{a o}(t)=\varphi^{T}(t) \theta+\epsilon(t) .
$$

Equation (6) is continuous-time model. An identification model is obtained by applying the numerical integration technique which is known as an effective approach for continuous-time model identification. For details, please refer to $[6]$.

Measurement data are sampled data of air-way pressure $P_{a o}(k)$, flow $F(k)$, volume $V(k)$, where $k(k=$ $1,2, \cdots, N)$ denotes sampling instant. $N$ define the data size. $T$ define the sampling period of data collection. Then, at time instant $t=k T$, integrate both sides of equation (7) over the interval $[(k-\ell) T, k T]$. Using the numerical integration technique that is proposed by Sagara et al, left side of equation (7) can be calculated as follows:

$$
y(k)=\int_{(k-\ell) T}^{k T} P_{a o}(\tau) d \tau \doteq \sum_{j=0}^{\ell} g_{j} P_{a o}(k-j),
$$

where, $\ell$ is a natural number that decides the window size of numerical integration. The coefficients $g_{i}(i=1,2, \cdots, \ell)$ are determined by formula of numerical integration. When the trapezoidal rule is taken, they are given as follows:

$$
\left\{\begin{array}{l}
g_{0}=g_{\ell}=T / 2, \\
g_{i}=T, \quad i=1,2, \cdots, \ell-1 .
\end{array}\right.
$$

As calculation of equation (8), data vector $\varphi(t)$ can be calculated by

$$
\phi(k)=\int_{(k-\ell) T}^{k T} \varphi(\tau) d \tau
$$




$$
=\left[\begin{array}{c}
-P_{a o}(k)+P_{a o}(k-\ell) \\
\ell \\
\sum_{j=0}^{\ell} g_{j} V(k-j) \\
\sum_{j=0}^{\ell} g_{j} V^{2}(k-j) \\
V(k)-V(k-\ell) \\
\ell \begin{array}{c}
\ell \\
\sum_{j=0} g_{j}|F(k-j)| F(k-j) \\
F(k)-F(k-\ell)
\end{array}
\end{array}\right] .
$$

Get together the approximation error $\Delta_{E}$ caused by numerical integration and the integral of original error term $\epsilon$ in $e(k)$. Namely, Let $e(k)$ be

$$
e(k)=\Delta_{E}+\int_{(k-\ell) T}^{k T} \epsilon(\tau) d \tau .
$$

Consequently, an identification model of discrete-time form is provided from equation (8), equation (10) and equation (11) as follows:

$$
y(k)=\phi^{T}(k) \theta+e(k) .
$$

From the measurements of air-way pressure $P_{a o}(k)$, flow $F(k)$, volume $V(k)$, calculates $y(k)$ and $\phi(k)$ at each time instant $k=\ell+1, \cdots, N$. And the vector equation is structured by them as follows:

$$
\mathbf{y}=\boldsymbol{\Phi} \theta+\mathbf{e},
$$

where, $\mathbf{y}=[y(N) \cdots y(\ell+1)]^{T}, \mathbf{\Phi}=[\phi(N) \cdots \phi(\ell+$ $1)]^{T}, \mathbf{e}=[e(N) \cdots e(\ell+1)]^{T}$.

The least squares estimate that minimizes the criterion function $J=\|\mathbf{y}-\mathbf{\Phi} \theta\|^{2}$ is given by

$$
\hat{\theta}=\left(\boldsymbol{\Phi}^{\mathbf{T}} \boldsymbol{\Phi}\right)^{-1} \boldsymbol{\Phi}^{\mathbf{T}} \mathbf{y} .
$$

Then, the function of consequent part about an arbitrary rule in fuzzy rules (4), in other words, the function of pulmonary elastance is estimated as

$$
\hat{f}_{E}(V)=\hat{k}_{1}+\hat{k}_{2} V .
$$

Executing the above-mentioned calculation on all fuzzy rules, fuzzy rules are made.

\subsection{Recursive Parameter Estimation}

In the previous technique, $\theta^{T}=\left[a_{1}, k_{1}, k_{2}, r_{1}, r_{2}, b_{2}\right]$ of respiratory system at each fuzzy rule was estimated, that is, $\theta$ of respiratory system at each fuzzy rule was estimated different value. Parameters of the pulmonary elastance $\left(\theta_{f}^{T}=\left[k_{1}, k_{2}\right]\right)$ are different in each fuzzy rules. However, it is considered that other parameters $\left(\theta_{o}^{T}=\left[a_{1}, r_{1}, r_{2}, b_{2}\right]\right)$ does not change in short time (one cycle breath). Thus, the previous estimation technique includes contradiction. Because parameters $\theta_{o}$ which does not change in short time are different value at each fuzzy rule, it is thought that different value have influence on the estimated pre- cision of the static $P-V$ curve. This study solves this contradiction. The algorithm of new method is as follows.

\section{(Step 1) Determination of $\theta_{o}$ value}

The problem mentioned above is caused by the pulmonary elastance fuzzy expression. When all parameters are estimated in each rule, $\theta_{o}$ take different value in each rule. Therefore, the value of the pulmonary elastance parameters $\theta_{f}$ and other parameters $\theta_{o}$ must be decided separately to prevent this problem. For a contradiction solution, first, the pulmonary elastance is assumed the third order polynomial expression $\left(f_{E}(V)=k_{1}+k_{2} V+k_{3} V^{2}+k_{4} V^{3}\right)$. Using this polynomial expression, the parameter estimation procedure in section $\mathbf{3 . 2}$ is carried out. An identification model of this step1 is as follows (16), (17), (18). Then, parameters $\theta_{o}=\left[a_{1}, r_{1}, r_{2}, b_{2}\right]$ are estimated using the least-squares method. For the detail procedure, please refer to a previous section. Estimated parameters $\theta_{o}$ are decided as value of one breathing cycle.

$$
\begin{aligned}
y(k)= & \phi^{T}(k) \theta+e(k) . \\
y(k)= & \int_{(k-\ell) T}^{K T} P_{a o}(\tau) d \tau \doteq \sum_{j=0}^{\ell} g_{j} P_{a o}(k-j) . \\
\phi(k)= & \int_{(k-\ell) T}^{K T} \varphi(\tau) d \tau \\
& {\left[\begin{array}{c}
-P_{a o}(k)+P_{a o}(k-\ell) \\
\ell \\
\sum_{j=0}^{\ell} g_{j} V(k-j) \\
\sum_{j=0}^{\ell} g_{j} V^{2}(k-j) \\
\sum_{j=0}^{\ell} g_{j} V^{3}(k-j) \\
\sum_{j=0}^{\ell} g_{j} V^{4}(k-j) \\
V(k)-V(k-\ell) \\
\ell \\
\sum_{j=0} g_{j}|F(k-j)| F(k-j) \\
F(k)-F(k-\ell)
\end{array}\right] . }
\end{aligned}
$$

(Step 2) Estimation of pulmonary elastance parameters $\theta_{f}$

Using estimated $\theta_{o}$ in step1, parameters of pulmonary elastance expression $f_{E i}(V)=k_{1 i}+k_{2 i} V(i=$ small,medium, big) in each fuzzy rule are estimated. An identification models is as follows.

$$
y(k)=\phi^{T}(k) \theta_{f}+e(k)
$$




$$
\begin{aligned}
y(k) & =\int_{(k-\ell) T}^{K T} P_{a o}(\tau) d \tau-a_{1} \int_{(k-\ell) T}^{K T}-\dot{P_{a o}}(\tau) d \tau \\
- & r_{1} \int_{(k-\ell) T}^{K T} F(\tau) d \tau-r_{2} \int_{(k-\ell) T}^{K T}|F(\tau)| F(\tau) d \tau \\
- & b_{2} \int_{(k-\ell) T}^{K T} \dot{F}(\tau) d \tau \\
\phi(k) & =\int_{(k-\ell) T}^{K T} \varphi(\tau) d \tau \\
& {\left[\begin{array}{l}
\ell \\
\sum_{j=0}^{\ell} g_{j} V(k-j) \\
\sum_{j=0}^{\ell} g_{j} V^{2}(k-j)
\end{array}\right] }
\end{aligned}
$$

(Step 3) Estimation of pulmonary elastance $f_{E}(V)$

Using the estimated parameters in Step 2 and fuzzy rule (4), the pulmonary elastance is estimated by equation (5).

(Step 4) New parameter $\theta_{o}$ estimation using estimated pulmonary elastance $f_{E}(V)$

Estimated pulmonary elastance values in Step 3 is available in the estimation of a new parameter $\theta_{o}$. Using estimated pulmonary elastance values, pulmonary internal pressures $P_{\ell}$ of each volume is estimated.

$$
\hat{P}_{\ell}=\hat{f}_{E}\left(V, \theta_{f}\right) V
$$

When equation (22) is substituted for equation (2) of respiratory system model, respiratory system model is as follows:

$$
\begin{aligned}
P_{a o}(t)+a_{1} \dot{P}_{a o}(t)= & \hat{P}_{\ell}+r_{1} \dot{V}(t)+r_{2}|F(t)| F(t) \\
& +b_{2} \dot{F}(t)+\epsilon(t) .
\end{aligned}
$$

Using the numerical integration technique, an identification models is as follows:

$$
\begin{aligned}
y(k) & =\phi^{T}(k) \theta_{o}+e(k) \\
y(k) & =\sum_{j=0}^{\ell} g_{j} P_{a o}(k-j)-\sum_{j=0}^{\ell} g_{j} \hat{P}_{\ell}(k-j) \\
\phi(k) & =\int_{(k-\ell) T}^{K T} \varphi(\tau) d \tau \\
& {\left[\begin{array}{c}
-P_{a o}(k)+P_{a o}(k-\ell) \\
V(k)-V(k-\ell) \\
\sum_{j=0} g_{j}|F(k-j)| F(k-j) \\
F(k)-F(k-\ell)
\end{array}\right] . }
\end{aligned}
$$

Using the least-squares method, the new parameters of other terms $\left(\theta_{o}\right)$ is estimated. Then, return to Step
2 and Step 3, new static $P-V$ curve is estimated using fuzzy logic. If fuzzy expression is superior to the third order polynomial expression for pulmonary elastance $f_{E}(V)$, the estimated new static $P-V$ curve will be improved.

\section{Setting a Ventilation Condition of the Artificial Respirator by Fuzzy Logic}

In this section, we introduce technique that set the ventilation condition of the artificial respirator by estimated static $P-V$ curve in previous section. For the existing setting method, technique using the rotational transform exists[1]. However, this method is applicable to only general static P-V curve. The setting method that can fit various form of static P-V curve is necessary. Therefore, we suggest ventilation condition setting method using a fuzzy logic.

The fuzzy estimation is carried out by the composition of fuzzy rule. In suggestion estimation technique, the fuzzy rule is constructed by three rules. Each rule is similar in "part of starting $(V=$ small $)$ " "part of stability $(V=$ medium)" "part of saturation $(V=b i g)$ " that characterize the static $P-V$ curve. In addition, generally, the air-way pressure limit value of the artificial respirator is set by the saturated starting point of the static $P-V$ curve. On the basis of the two point mentioned above, if a fuzzy rule of " $V=b i g$ " and a fuzzy rule of " $V=$ medium" are appropriately similar with the characteristic of the static $P-V$ curve, it is thought that the saturated starting point is near a point that is reversing estimated pulmonary elastance value of two rules.(Please refer to a Fig. 2) We use the mean-square error values of inspection pressure data and estimated pressure data as a quantitative evaluation in this study. Therefore, the estimated pulmonary internal pressure $P_{\ell}$ at above point is set as the air-way pressure limit value $P_{m}$. Please note that this method will fail if each fuzzy rule is not similar to character the static $P-V$ curve, that is, the mean-square error value take a big value. Therefore, the composition of the fuzzy rule is important.

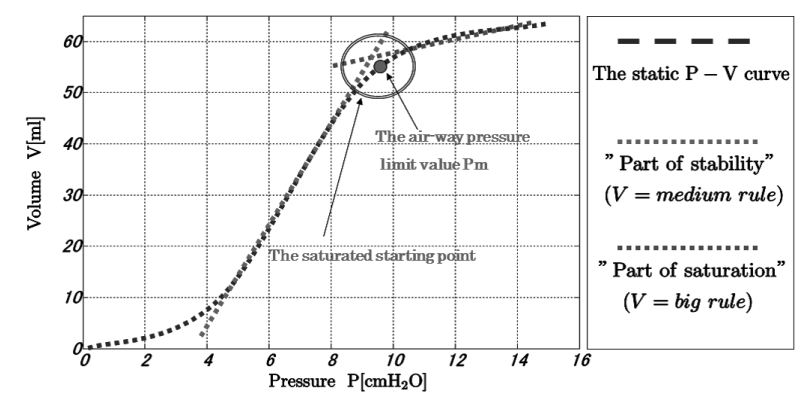

Fig. 2 Image of deciding the air-way pressure limit value using fuzzy logic

An advantage of this technique is to be able to reuse the value of estimated pulmonary elastance. Thus, 
this technique does not need a computational effort in comparison with previous technique. In this way, the fuzzy logic is not only suitable for the nonlinear function expression of the pulmonary elastance, but also effective for setting a ventilation condition of the artificial respirator.

\section{An Example of Estimation}

In this section, an experimental result is shown to estimate the static $P-V$ curve with real clnical data and to set the ventilation condition using estimated static $P-V$ curve.

Used patient data for estimation is data of one cycle breath. As a result of trial and error, ranges of the fuzzy variables (small, medium, big) are set by manual operation. Fig. 3 is membership functions of fuzzy variables in this experiment. Algorithm is iterated 100 times at paramters $\theta_{o}$ estimation procedure (step 1 or step 4 ), parameters $\theta_{f}$ estimation procedure (step 2) and estimation procedure of the pulmonary elastance $f_{E}(V)$ using the fuzzy reasoning method (step 3). We researched an estimation precision of the estimated static $P-V$ curve for each iteration number of times. Fig. 4 and Table 1 is mean-square error values of estimated static $P-V$ curve to inspection data for each iteration number of times. For these results, the third order polynomial expression is used at step 1. Fig. 5 and Table 2 is results of using the fourth order polynomial expression at step1. Whenever suggestion technique is iterated, the estimation precision of the estimated static $P-V$ curve is improved. However, the improvement value of the estimation precision decreases at each iteration. Fig. 6 is experimental results that estimated static $P-V$ curve with real clinical data of artificial respiration at algorithm 100 times iteration. The circle points are quasi-static elastance $P-V$ points for inspection. Then, cross point is setting point for the air-way pressure limit value $P_{m}$ that decided using fuzzy logic. It is shown that the estimated static $P-V$ curve passes near of inspection data.

Table 1 Mean-square error values of estimated static $P-V$ curve to inspection data for each iteration number of times (Algorithm step1 used the third order polynomial expression)

\begin{tabular}{|c|c|}
\hline Iteration & Mean-square error \\
\hline 1 & $0.319004\left[\mathbf{c m H}_{\mathbf{2}} \mathbf{O}\right]$ \\
\hline 2 & $0.318297\left[\mathbf{c m H}_{\mathbf{2}} \mathbf{O}\right]$ \\
\hline 3 & $0.317772\left[\mathbf{c m H}_{\mathbf{2}} \mathbf{O}\right]$ \\
\hline 4 & $0.317379\left[\mathbf{c m H}_{\mathbf{2}} \mathbf{O}\right]$ \\
\hline 5 & $0.317084\left[\mathbf{c m H}_{\mathbf{2}} \mathbf{O}\right]$ \\
\hline$\vdots$ & $\vdots$ \\
\hline 99 & $0.316176\left[\mathbf{c m H}_{\mathbf{2}} \mathbf{O}\right]$ \\
\hline 100 & $0.316176\left[\mathbf{c m H}_{\mathbf{2}} \mathbf{O}\right]$ \\
\hline
\end{tabular}

Table 2 Mean-square error values of estimated static $P-V$ curve to inspection data for each iteration number of times (Algorithm step1 used the fourth order polynomial expression)

\begin{tabular}{|c|c|}
\hline Iteration & Mean-square error \\
\hline 1 & $0.317539\left[\mathbf{c m H}_{\mathbf{2}} \mathbf{O}\right]$ \\
\hline 2 & $0.317189\left[\mathbf{c m} \mathbf{H}_{\mathbf{2}} \mathbf{O}\right]$ \\
\hline 3 & $0.316939\left[\mathbf{c m} \mathbf{H}_{\mathbf{2}} \mathbf{O}\right]$ \\
\hline 4 & $0.316752\left[\mathbf{c m} \mathbf{H}_{\mathbf{2}} \mathbf{O}\right]$ \\
\hline 5 & $0.316611\left[\mathbf{c m} \mathbf{H}_{\mathbf{2}} \mathbf{O}\right]$ \\
\hline$\vdots$ & $\vdots$ \\
\hline 99 & $0.316176\left[\mathbf{c m H}_{\mathbf{2}} \mathbf{O}\right]$ \\
\hline 100 & $0.316176\left[\mathbf{c m H}_{\mathbf{2}} \mathbf{O}\right]$ \\
\hline
\end{tabular}

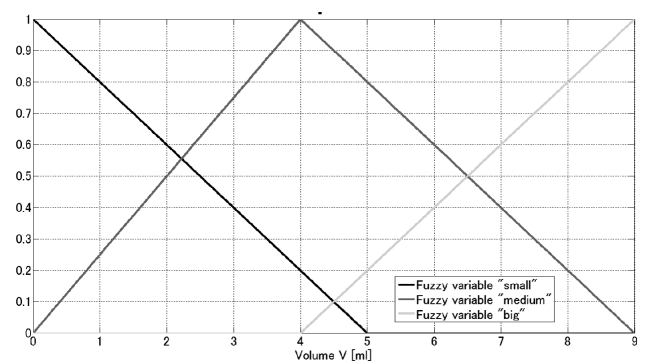

Fig. 3 Membership functions of fuzzy variables

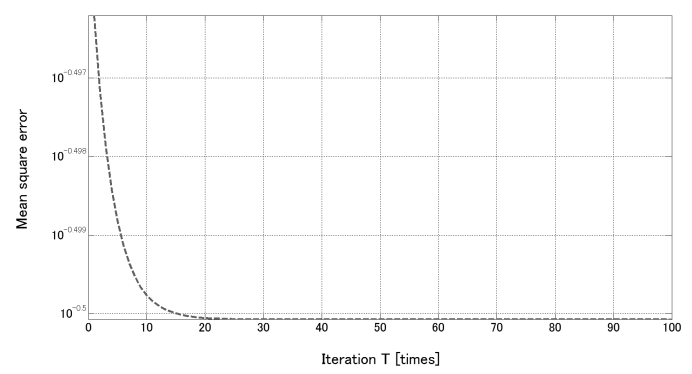

Fig. 4 Variation result of Mean-square error values (Algorithm step1 used the third order polynomial expression)

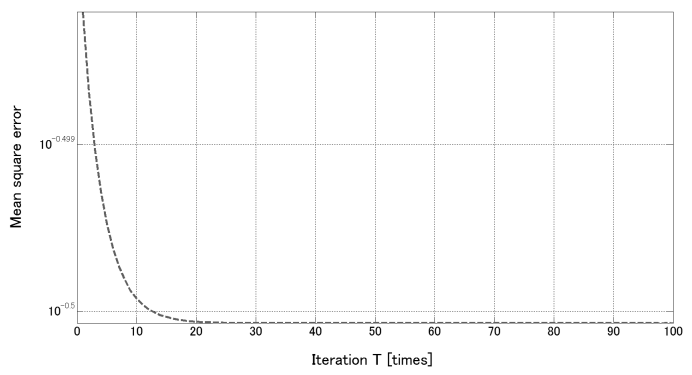

Fig. 5 Variation result of Mean-square error values (Algorithm step1 used the fourth order polynomial expression)

\section{Conclusions}

The pulmonary elastance is one of the important features of lung, and it is a basis for deciding the 


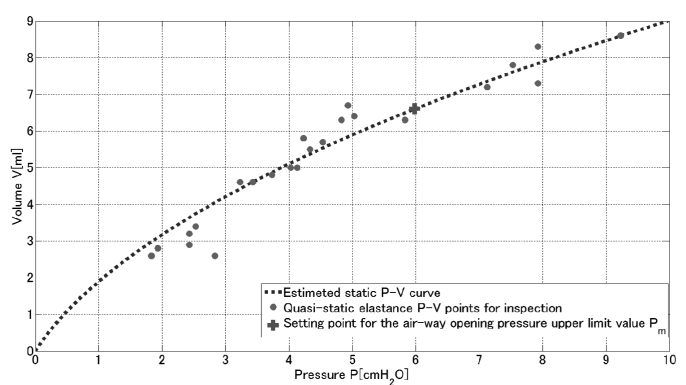

Fig. 6 Estimated static $P-V$ curve(dotted line) and setting point for the air-way pressure limit value $P_{m}$

air-way pressure limit value. In this study, a new estimation method of pulmonary elastance based on fuzzy logic is proposed.

As a result of estimated pulmonary elastance with real clinical data of artificial respiration, we can draw the static $P-V$ curve that passes near of inspectional data points. Whenever a recursive estimation technique is iterated, the estimation precision of the estimated static $P-V$ curve is improved. However, ranges of the fuzzy variables are set by manual operation in this experiment. We aim at the combination of this technique and the ant colony optimization method that is optimization algorithm of the fuzzy variables[7] in the future.

\section{References}

[1] S. Kanae: The setting of the ventilation condition of the artificial respiration and the modeling of respiratory system; Measurement and Control, Vol. 49, No. 7, pp. 485-488 (2010)

[2] K. Muramatsu, K. Yukitake, M. Nakamura, I. Matsumoto and Y. Motohiro: Monitoring of nonlinear respiratory elastance using a multiple linear regression analysis; European Respiratory Journal 17, pp. 1158-1166 (2001)

[3] S. Kanae, K. Muramatsu, Z. J. Yang and K. Wada:
Modeling of respiration and estimation of pulmonary elastance; 2004 ASCC, pp. 648-651 (2004)

[4] M. Sugeno: Fuzzy Control, Daily publication industry newspaper publishing company (1990)

[5] M. Nakamichi, S. Kanae, Z. J. Yang and K. Wada: Estimation of pulmonary elastance fuzzy model by data combination of two respiration phases; The 2012 UKACC International Conference on Control, pp. 953-957 (2012)

[6] S. Sagara and Z. Zhao: Numerical integration approach to on-line identification of continuous-time systems; Automatica, Vol. 26, pp. 63-74 (1990)

[7] S. Kanae and M. Nakamichi: An ant colony optimization method for fuzzy membership functions and its application to estimate the pulmonary elastance; The 32th CCC, pp. 8056-8060 (2013)

\section{Authors}

Masanori NAKAMICHI (Member)

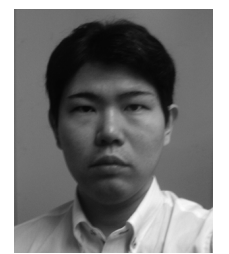

Masanori NAKAMICHI was born in 1981. He is a Ph.D. Degree from Fukui University of Technology. He works as a lecturer in Fukui University of Technology, from 2014-present.

\section{Shunshoku KanaE (Member)}

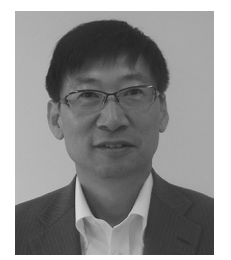

Shunshoku KANAE was born in 1961. He received the Dr. Eng. Degree from Kyushu University in 1995. He is currently a professor in the department of electric, electronic and computer engineering, Fukui University of Technology. His research interests include system identification, mechatronics system control and medical engineering. 